\title{
The elastic-plastic response of aluminum films to ultrafast laser-generated shocks
}

\author{
V. H. Whitley, ${ }^{1, a)}$ S. D. McGrane, ${ }^{1}$ D. E. Eakins, ${ }^{1}$ C. A. Bolme, ${ }^{1}$ D. S. Moore,${ }^{1}$ and \\ J. F. Bingert ${ }^{2}$ \\ ${ }^{1}$ Shock and Detonation Physics-WX9, Los Alamos National Laboratory, Los Alamos, \\ New Mexico 87545, USA \\ ${ }^{2}$ Structure/Property Relations-MST8, Los Alamos National Laboratory, Los Alamos, \\ New Mexico 87545, USA
}

(Received 22 July 2010; accepted 29 September 2010; published online 5 January 2011)

\begin{abstract}
We present the free surface response of 2, 5, and $8 \mu \mathrm{m}$ aluminum films to shocks generated from chirped ultrafast lasers. We find two distinct steps to the measured free surface velocity that indicate a separation of the faster elastic wave from the slower plastic wave. We resolve the separation of the two waves to times as short as $20 \mathrm{ps}$. We measured peak elastic free surface velocities as high as 1.4 $\mathrm{km} / \mathrm{s}$ corresponding to elastic stresses of $12 \mathrm{GPa}$. The elastic waves rapidly decay with increasing sample thickness. The magnitude of both the elastic wave and the plastic wave and the temporal separation between them was strongly dependent on the incident laser drive energy. (C) 2011 American Institute of Physics. [doi:10.1063/1.3506696]
\end{abstract}

\section{INTRODUCTION}

The elastic and plastic stress waves of aluminum generated under shock loading have been studied extensively. Previous work on thick aluminum samples, on the order of centimeter and larger, showed small elastic stress amplitudes of $\sim 0.1 \mathrm{GPa}$ and little elastic attenuation. ${ }^{1-4}$ Based on these measurements, aluminum was considered to have a rateindependent elastic stress as a function of thickness.

Though the elastic stress was considered rateindependent in thick samples, several experiments have revealed a decay of the elastic stress in aluminum samples less than a centimeter in thickness. Arvidsson et al. ${ }^{3}$ measured the decay of 1060 aluminum from $0.106 \mathrm{GPa}$ in $1.38 \mathrm{~mm}$ thick samples to $0.053 \mathrm{GPa}$ in $9.68 \mathrm{~mm}$ thick samples. Winey et $a l .{ }^{5}$ measured the decay of 1050 aluminum from $0.51 \mathrm{GPa}$ in $77 \mu \mathrm{m}$ thick samples to $0.19 \mathrm{GPa}$ in $1045 \mu \mathrm{m}$ thick samples. Gupta et al. ${ }^{6}$ measured the decay, of $99.999 \%$ pure aluminum from $0.95 \mathrm{GPa}$ in $43 \mu \mathrm{m}$ thick samples to 0.46 $\mathrm{GPa}$ in $183 \mu \mathrm{m}$ thick samples. Based on these decay values, Winey ${ }^{5}$ and Gupta $^{6}$ suggested that the elastic wave amplitudes near the impact surface were large but attenuated rapidly. Smith et al. ${ }^{7}$ measured the elastic precursors in aluminum films ranging from $10-30 \mu \mathrm{m}$ using a shockless compression technique. They measured elastic stresses up to 2.7 GPa that did not decay with increasing film thickness.

Below $10 \mu \mathrm{m}$ thickness, the elastic-plastic response of aluminum to shocks has not been measured. Both the magnitude of the elastic stress and the onset of separation between the elastic and plastic waves are unknown. Prior work using compressed $\sim 150$ fs duration laser pulses have generated shocks in films of $0.250-1.0 \mu \mathrm{m}$ thickness and observed only a single wave. ${ }^{8-11}$ Molecular dynamic models on

\footnotetext{
${ }^{a)}$ Electronic mail: vwhitley@lanl.gov.
}

metals, however, suggest that the transition from elastic to plastic deformation response may occur on micron length scales and picosecond time scales. ${ }^{12-14}$

Using a shaped chirped-pulse amplified ultrafast laser to generate supported shocks (to be discussed later), we have successfully generated and measured both elastic and plastic waves in aluminum films ranging from $2-8 \mu \mathrm{m}$ thick. The following work reports the highest elastic amplitude measured in aluminum to date and resolves the separation of the faster elastic wave from the plastic wave to times as short as $20 \mathrm{ps}$ in films as thin as $2 \mu \mathrm{m}$.

\section{EXPERIMENTAL METHOD}

For these experiments, thin aluminum films were deposited onto $120 \mu \mathrm{m}$ thick glass cover slips. The aluminum films were obtained from two different sources. Samples of 2,5 , and $8 \mu \mathrm{m}$ thickness were vapor deposited by Berliner Glas KGaA. For comparison, a second set of samples were prepared at Los Alamos using thermal evaporative vapor deposition. Aluminum was deposited onto UV-ozone cleaned glass cover slips to desired thickness as measured by an in situ quartz crystal microbalance. Deposition rates for these samples were nominally $20 \mathrm{~nm} / \mathrm{s}$. The substrates were rotated during deposition to ensure uniform thickness across the sample.

Starting material for deposition ranged from 99\%99.999\% purity. The elastic-plastic response of our films did not vary significantly with start material purity, suggesting that impurity levels below $1 \%$ in vapor deposited samples have little effect on the elastic-plastic behavior. The final films produced by Berliner Glas and the Los Alamos films had highly reflective surfaces.

Electron backscatter diffraction (EBSD) was used to characterize selected regions from the $2 \mu \mathrm{m}$ thick Berliner Glas samples. Although the scan was collected using a $20 \mathrm{~nm}$ step size, some of the finer structure may not have been 

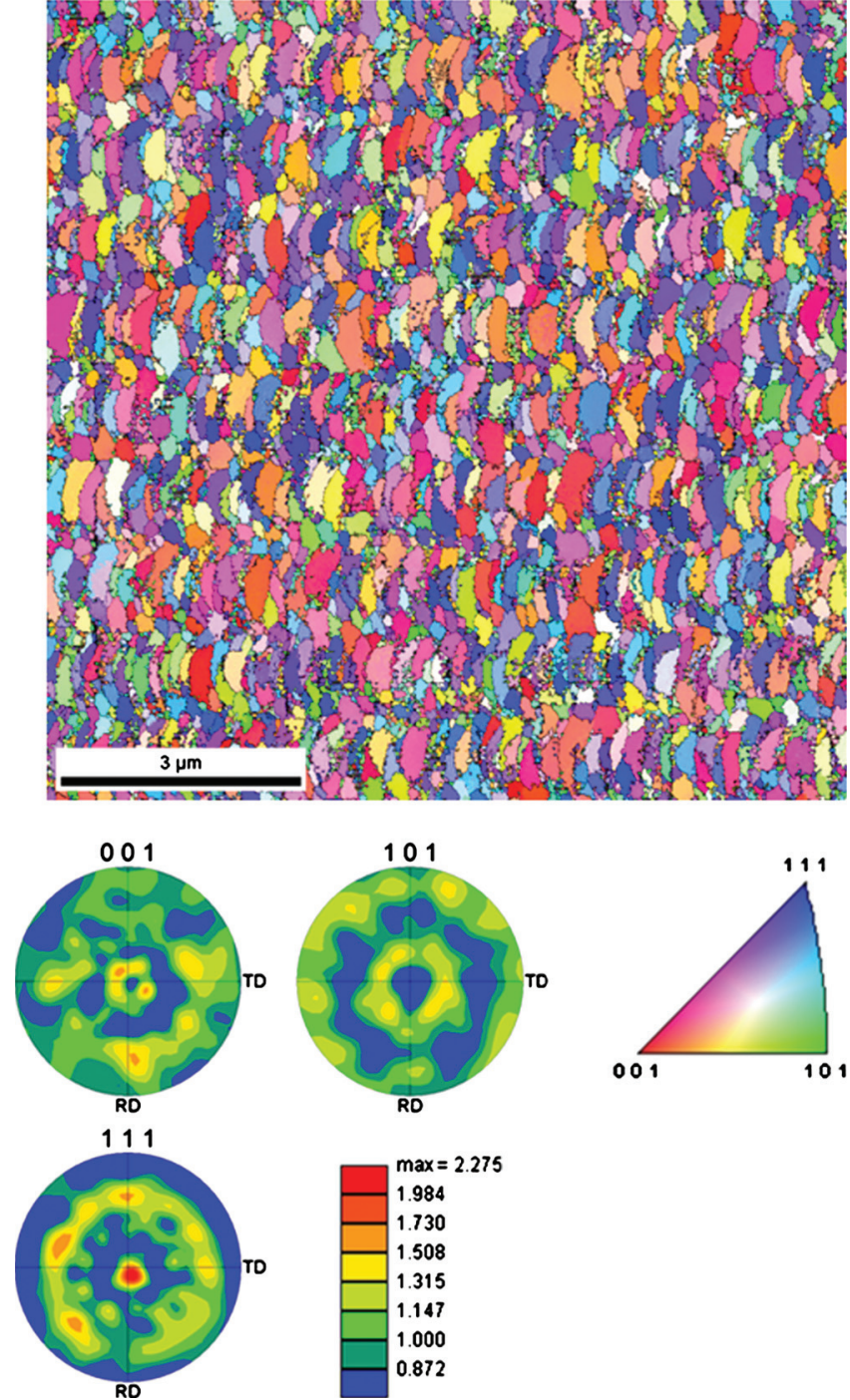

FIG. 1. (Color) EBSD scan results in the form of a crystal direction map and related pole figures from representative $2 \mu \mathrm{m}$ aluminum film used in these experiments. Scan collected at $20 \mathrm{~nm}$ step size.

captured. Figure 1 shows an EBSD crystal-direction map with respect to the sample normal from a representative region of the film, along with pole figures extracted from the orientation data. The structure consisted of approximately $500 \mathrm{~nm} \times 100 \mathrm{~nm}$ platelets with a moderate $\langle 111\rangle /\langle 001\rangle$ duplex fiber texture.

A chirped pulse amplified Ti:sapphire laser was used both to shock the aluminum film and to probe the free surface motion of the aluminum after shock breakout. Details of the technique used for the free surface measurements were published by Bolme et al. ${ }^{15}$ and details for the spectral shaping of the shock drive pulse were published by McGrane et $a l .{ }^{16}$ For these experiments, the laser was focused through the glass to an $\sim 100 \mu \mathrm{m}$ spot at the aluminum film to generate the shock. Since the energy of the drive pulse was Gaussian shaped across the spatial dimension, we present data from an approximately $5 \mu \mathrm{m}$ spatial region in the center of the shock, where the shock was traveling normal to the surface of the aluminum.

The incident angle of the probe beam was $30^{\circ} \pm 1^{\circ}$. Both s- and p-polarizations were measured. Our diagnostic
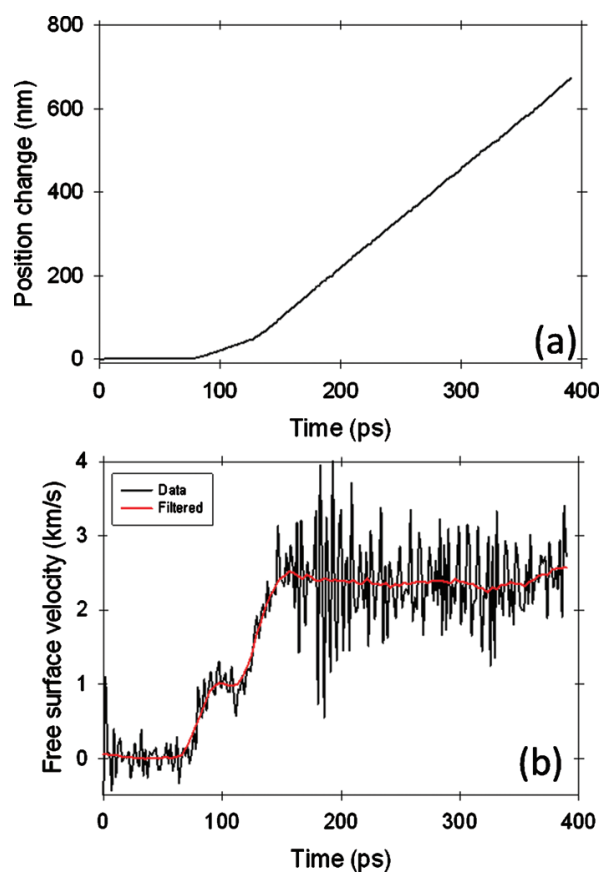

FIG. 2. (Color) (a) Measured free surface displacement of $8 \mu \mathrm{m}$ thick Al film. (b) Free surface velocity of aluminum film showing the noise and the results of filtering the data.

technique monitors the position of the free surface, which we converted to free surface velocity by taking a point-to-point numerical derivative of the data. The results of converting the displacement measurement, shown in Fig. 2(a), into a free surface velocity measurement are shown in Fig. 2(b). Even though the surface position measurements were very smooth in displacement space, the differentiation amplified high-frequency noise in the free surface velocity. This highfrequency noise was low-pass filtered and is shown overlaid with the unfiltered free surface velocity. Unfortunately, the low-pass filter broadened features that were faster than $20 \mathrm{ps.}$ While the temporal resolution of the free surface measurement was $\sim 5 \mathrm{ps}$, the rise times and the separations between the elastic and plastic waves cannot be resolved to times shorter than $20 \mathrm{ps}$ in the filtered data. The low-pass filter also averaged the leading edge of the elastic wave. Since some of the measured data show a peak at the leading edge of the elastic wave, followed by a pullback, the low-pass filtered data can result in a lower reported peak elastic-free surface velocities.

The temporal profile of the shock drive was determined using a cross correlation frequency resolved optical gating method, as described by Linden et al. ${ }^{17}$ The laser pulse used to generate the shock had a rise time of $5 \mathrm{ps}$, followed by a relatively constant energy for $\sim 300$ ps. The temporal profile of the shock drive pulse is shown in Fig. 3. We assume the shock to be supported as long as energy from the laser is flowing into the shock drive. Assuming the shock velocity through the aluminum films to be maximally $10 \mathrm{~km} / \mathrm{s}$, the shocks were fully supported through $3 \mu \mathrm{m}$ of material. In the thickest samples, $5 \mu \mathrm{m}$ and $8 \mu \mathrm{m}$ respectively, the shocks generated in the aluminum were not fully supported for the full duration of the experiment. 


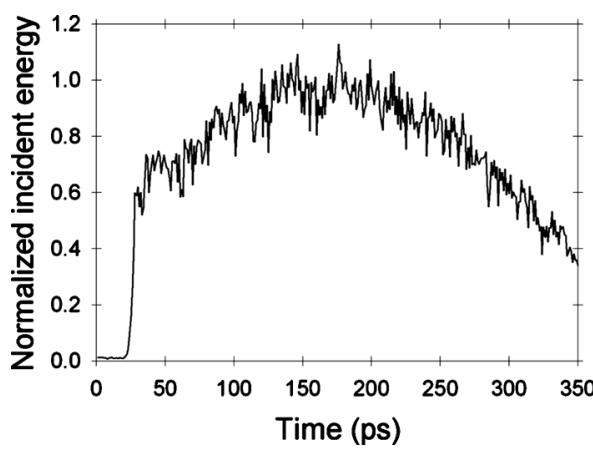

FIG. 3. Temporal profile of shock drive pulse.

\section{RESULTS AND DISCUSSION}

Figure 2(a) shows the resulting surface displacement during a shock as a function of time. Initially, the surface was stationary for a period of time before we saw any displacement. Upon stress breakout, the free surface exhibits motion characterized by two different displacement rates separated by a distinct cusp. The slopes of both lines and the temporal location of the cusp were strongly dependent on the incident energy in the drive pulse. The filtered free surface velocity in Fig. 2(b) clearly demonstrates two steps similar to elastic-plastic separations found in planar impact experiments of thicker aluminum. These two steps are due to the same elastic-plastic wave separation found in bulk aluminum but on length scales of microns and time scales of picoseconds.

These elastic and plastic waves could be reproducibly measured across a variety of samples, film thicknesses and drive energies. The results of six adjacent shocks generated in $5 \mu \mathrm{m}$ thick aluminum using an incident laser energy of $2.0 \mathrm{~mJ}$ for all six shocks are shown in Fig. 4. We measured similar separation times between the elastic and plastic waves and similar free surface velocities for the elastic and plastic waves even though the data were measured at six different spots on the sample. Shot-to-shot fluctuations in the energy of the laser drive, estimated at $\pm 10 \%$, were largely responsible for the variance in the data shown in Fig. 4. Measurements taken at different laser energies or on different samples showed similar shot-to-shot consistency in the measured elastic and plastic profiles.

Figure 5 summarizes a series of experiments performed on a single thickness of aluminum over a range of input

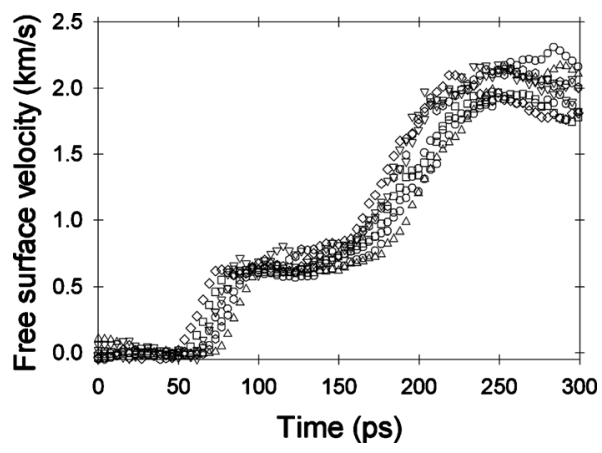

FIG. 4. Free surface velocity profiles of six different laser-generated shocks on $5 \mu \mathrm{m}$ thick aluminum using $2.0 \mathrm{~mJ}$ incident drive energy.

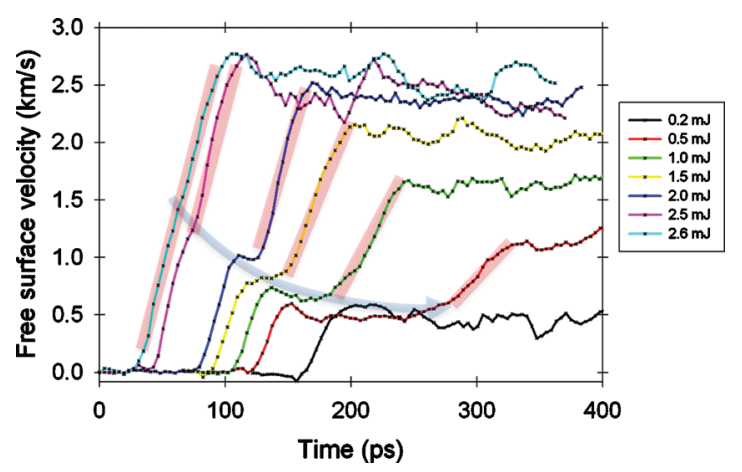

FIG. 5. (Color) Changes in the elastic and plastic waves in $8 \mu \mathrm{m} \mathrm{Al} \mathrm{films}$ as a function of incident drive energy. The blue line highlights the decay of the elastic wave with decreasing incident drive energy. The red line highlights the change in slope of the plastic wave as a function of drive energy.

stresses. We present the data for the $8 \mu \mathrm{m}$ film but we measured the response of all three film thicknesses to various input stresses. Even though the shock is not fully supported through the $8 \mu \mathrm{m}$ sample, the temporal separation between the elastic and plastic waves was greater than the 2 and $5 \mu \mathrm{m}$ samples. It should be noted that both the 2 and $5 \mu \mathrm{m}$ samples showed similar elastic-plastic wave separation, albeit with shorter separation times between the elastic and plastic wave.

Drive energies ranged from 0.2 to $2.6 \mathrm{~mJ}$ in these experiments; increasing laser energy drives increasingly stronger shocks into the aluminum film. Elastic stresses were calculated using the maximum particle velocity (assuming particle velocity is half the measured free surface velocity) and a longitudinal sound speed for aluminum 1050 of 6.41 $\mathrm{km} / \mathrm{s}{ }^{5}$ Plastic stresses were calculated using the Hugoniot of aluminum determined by Mitchell and Nellis. ${ }^{18}$ The drive energies used in these experiments correlated with shocks with nominal pressures of 4-24 GPa in the films. Two prominent trends are found in Fig. 5. The first trend is shown by the blue line. Here, the initial free surface motion of the highest drive energy $(2.6 \mathrm{~mJ})$ began $130 \mathrm{ps}$ sooner than the free surface motion at the lowest drive energy $(0.2 \mathrm{~mJ})$ and the magnitude of the initial-free surface velocity increased with increasing drive stress. The second prominent trend shown in Fig. 5 is marked by the red regions. The magnitude and delay of late time-free surface velocity was strongly dependent on the strength of the shock traversing the film. At the lowest drive energy, the second step was either not present or sufficiently retarded that it did not appear within the 300 ps of subsequent motion that we monitored. As progressively stronger drive energies were used for shock generation, the magnitude of the free surface velocity increased and the delay between the elastic and plastic wave decreased. The slope increased with increasing drive energy until it approached that of the initial slope. At $2.5 \mathrm{~mJ}$ drive energy, the two waves have very nearly merged into a single wave, temporally separated by nominally $20 \mathrm{ps}$. At $2.6 \mathrm{~mJ}$, we no longer resolve a temporal separation between the elastic and plastic waves.

Figure 6 shows the decay of the elastic wave as a function of sample thickness. For these experiments, we used a fixed laser energy and identical focal area for the laser drive 


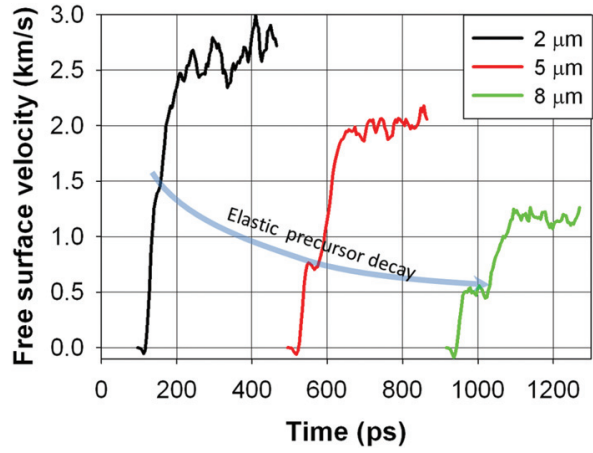

FIG. 6. (Color) Elastic wave decay as a function of thickness in aluminum. The same incident laser drive energy was used for all data.

but varied the thickness of the aluminum film. The initial input stress was similar for all thicknesses. The measured particle velocity corresponding to the elastic wave decreased from $1.4 \mathrm{~km} / \mathrm{s}$ in the $2 \mu \mathrm{m}$ sample to $0.5 \mathrm{~km} / \mathrm{s}$ in the $8 \mu \mathrm{m}$ sample. The delay of the plastic wave increased in separation from the elastic wave as it traversed more material, from 20 ps in the $2 \mu \mathrm{m}$ film to $65 \mathrm{ps}$ in the $8 \mu \mathrm{m}$ film. The final stress state also decreased as a function of increasing sample thickness. As discussed previously, the 300 ps drive duration of our chirped laser pulse is insufficient to fully support shocks through sample thicknesses much greater than $3 \mu \mathrm{m}$. Thus the final stress state measured in both the 5 and $8 \mu \mathrm{m}$ films had begun to decay before reaching the free surface.

A comparison of the initial elastic response across the 2, 5 , and $8 \mu \mathrm{m}$ samples revealed a consistent $\sim 20$ ps rise time. Taking into consideration the low-pass filter broadening, our measured rise time is within reasonable agreement with prior measurements of 4-6 ps in thin aluminum films using femtosecond laser driven shocks. ${ }^{9-11}$

The elastic and plastic waves, shown in Figs. 5 and 6, exhibit a similar behavior on this sub-10 $\mu \mathrm{m}$ scale to stresses in aluminum on the order of hundreds of microns to centimeters. We measure an elastic precursor that (a) decays with increasing aluminum thickness and (b) decays with decreasing input stress. The magnitude of the measured elastic precursor, however, is orders of magnitude stronger than that measured in millimeter sized samples and is a factor of 5 larger than the previously strongest elastic precursors measured on $10 \mu \mathrm{m}$ thick films of aluminum. ${ }^{7}$ The amplitude of the elastic wave should increase exponentially as samples get thinner, ${ }^{19,20}$ thus we should be measuring much higher elastic waves with these comparatively thin samples.

\section{CONCLUSIONS}

We have presented experimental results on the response of 2-8 $\mu \mathrm{m}$ thick aluminum films to strong laser-generated shocks. We conclusively measured both an elastic and plastic wave at the free surface of our aluminum films. The peak resolved elastic stress reached values as high as $12 \mathrm{GPa}-$ almost five times higher than the largest values reported to date.

The responses of these thin metal films to lasergenerated shocks were similar to planar impact experiments, albeit at a much shorter time scale. The peak elastic wave rapidly decays with increasing sample thickness, from 12 $\mathrm{GPa}$ in $2 \mu \mathrm{m}$ thick films to $4.3 \mathrm{GPa}$ in $8 \mu \mathrm{m}$ thick films. The magnitude of both the elastic wave and the plastic wave, and the temporal separation between them was strongly dependent on the incident laser drive energy. At the lowest laser energies, only an elastic wave was measured. As the laser energy was increased, the separation between the elastic wave and the plastic wave decreased. At the highest energies used, we could no longer distinguish the elastic from the plastic wave.

These are the first experimental observations of both elastic and plastic waves developing within $2 \mu \mathrm{m}$ of the shock surface and temporally separated by times as short as 20 ps. These time and length scales are well suited for comparison with molecular dynamic calculations of shocked materials. Future work will attempt to experimentally verify predictions about the very early time shock behavior of materials.

\section{ACKNOWLEDGMENTS}

We gratefully acknowledge the support of the U.S. Department of Energy through the LANL/LDRD Program for this work. The authors would like to thank Ron Rabie, Paulo Rigg, and Brian Jensen for valuable discussions and suggestions.

${ }^{1}$ G. R. Fowles, J. Appl. Phys. 32, 1475 (1961).

${ }^{2}$ J. N. Johnson and L. M. Barker, J. Appl. Phys. 40, 4321 (1969).

${ }^{3}$ T. E. Arvidsson, Y. M. Gupta, and G. E. Duvall, J. Appl. Phys. 46, 4474 (1975).

${ }^{4}$ Y.-C. Hsu, C.-K. Chou, P. C. Liu, C. Chen, D. J. Yao, T. Chou, and K. N. Tu, J. Appl. Phys. 98, 033523 (2005).

${ }^{5}$ J. M. Winey, B. M. LaLone, P. B. Trivedi, and Y. M. Gupta, J. Appl. Phys. 106, 073508 (2009).

${ }^{6}$ Y. M. Gupta, J. M. Winey, P. B. Trivedi, B. M. LaLone, R. F. Smith, J. H. Eggert, and G. W. Collins, J. Appl. Phys. 105, 036107 (2009).

${ }^{7}$ R. F. Smith, J. H. Eggert, A. Jankowski, P. M. Celliers, M. J. Edwards, Y. M. Gupta, J. R. Asay, and G. W. Collins, Phys. Rev. Lett. 98, 065701 (2007).

${ }^{8}$ R. Evans, A. D. Badger, F. Falliès, M. Mahdieh, T. A. Hall, P. Audebert, J.-P. Geindre, J.-C. Gauthier, A. Mysyrowics, G. Grillon, and A. Antonetti, Phys. Rev. Lett. 77, 3359 (1996).

${ }^{9}$ K. T. Gahagan, D. S. Moore, D. J. Funk, R. L. Rabie, and S. J. Buelow, Phys. Rev. Lett. 85, 3205 (2000).

${ }^{10}$ D. J. Funk, D. S. Moore, K. T. Gahagan, S. J. Buelow, J. H. Reho, G. L. Fisher, and R. L. Rabie, Phys. Rev. B 64, 115114 (2001).

${ }^{11}$ K. T. Gahagan, D. S. Moore, D. J. Funk, J. H. Reho, and R. L. Rabie, J. Appl. Phys. 92, 3679 (2002).

${ }^{12}$ E. M. Bringa, K. Rosolankova, R. E. Rudd, B. A. Remington, J. S. Wark, M. Duchaineau, D. H. Kalantar, J. Hawreliak, and J. Belak, Nature Mater. 5, 805 (2006).

${ }^{13}$ R. Ravelo, B. L. Holian, and T. C. Germann, in Shock Compression of Condensed Matter, edited by M. L. Elert, W. T. Buttler, M. D. Furnish, W. W. Anderson, and W. G. Proud (American Institute of Physics, Nashville, Tennessee, 2009), Vol. 1195, pp. 825-828.

${ }^{14}$ T. C. Germann and K. Kadau, in Shock Compression of Condensed Matter, edited by M. L. Elert, W. T. Buttler, M. D. Furnish, W. W. Anderson, and W. G. Proud (American Institute of Physics, Nashville, Tennessee, 2009), Vol. 1195, pp. 1209-1212.

${ }^{15}$ C. A. Bolme, S. D. McGrane, D. S. Moore, and D. J. Funk, J. Appl. Phys. 102, 033513 (2007).

${ }^{16}$ S. D. McGrane, D. S. Moore, D. J. Funk, and R. L. Rabie, Appl. Phys. Lett. 80, 3919 (2002).

${ }^{17}$ S. Linden, H. Giessen, and J. Kuhl, Phys. Status Solidi B 206, 119 (1998).

${ }^{18}$ A. C. Mitchell and W. J. Nellis, J. Appl. Phys. 52, 3363 (1981).

${ }^{19}$ J. W. Taylor and M. H. Rice, J. Appl. Phys. 34, 364 (1963).

${ }^{20}$ J. R. Asay, G. R. Fowles, G. E. Durall, M. H. Miles, and R. F. Tinder, J. Appl. Phys. 43, 2132 (1972). 